\title{
LA DESCRIPCIÓN DEL FUNCIONAMIENTO DEL TÉRMINO EN EL DISCURSO ECONÓMICO: ESTUDIO COMPARATIVO DE LAS LENGUAS ESPAÑOLA, RUSA E INGLESA ${ }^{1}$
}

\author{
SVETLANA ABAKUMOVA / HERMENEGILDO LÓPEZ GONZÁLEZ \\ Universidad de Voronezh / Universidad de León
}

\begin{abstract}
Resumen
El artículo está dedicado al problema del funcionamiento de los términos que describen la situación de la crisis económica y los aspectos ligados a la misma, en la variante oral y escrita del discurso económico. El análisis del discurso económico nos ofrece la posibilidad de verificar el funcionamiento activo del término, lo que no se refleja en los diccionarios.
\end{abstract}

Palabras clave: término, terminología, discurso, discurso económico, crisis económica, recesión, reconstitución (de la economía, de la industria).

\begin{abstract}
The article is focused on the problem of the way terms, describing economic crisis and other aspects of this topic, function in written and spoken variants of economic discourse. Economic discourse gives the opportunity to observe the way terms function in reality, what is practically impossible to do with the help of only dictionaries.
\end{abstract}

Key words: term, terminology, discourse, economic discourse, economic crisis, recession, economic recovery.

La actualidad de la presente investigación viene determinada por la importancia y la influencia que los aspectos económicos tienen en la sociedad actual: paralelamente, o como consecuencia de ello, se ha producido un enorme desarrollo de la comunicación oficial, de las revistas y periódicos especializados, etc., es decir del propio discurso económico.

Durante las últimas décadas y de manera especialmente significativa desde los primeros intentos de integración de Rusia en la economía de mercado y en un contexto político y económico global, esta tendencia se ha acelerado de modo evidente, contribuyendo así al proceso de enriquecimiento del vocabulario ruso. Entre estos nuevos aportes léxicos predominan, por tanto, los relativos a los campos económico y financiero. No estamos, por otra parte, ante una novedad digna de mención, puesto

\footnotetext{
${ }^{1}$ Universidad de León. Correo: hlopg@unileon.es. Recibido: 29-01-2011. Aceptado: 14-03-2011.
} 
que el fenómeno pude ser detectado en el resto de las lenguas de cultura; no podemos obviar que la economía y las relaciones económicas pertenecen a un campo que reacciona rápidamente a las renovaciones e inovaciones; por lo mismo, el léxico debe, necesariamente, ir cambiando y adaptándose de manera constante a nuevos tiempos y nuevos retos.

Desde el punto de vista de su propia formación, la terminología económica actual se divide en una serie de campos. Los investigadores destacan, sin embargo, una constante: un sustrato considerable de unidades léxicas que representan un fondo sostenido de terminología económica y que refleja las categorías y conceptos macroeconómicos básicos (Kandelaki 1977) y Nelubin 2003). El grupo más numeroso que hoy representa una serie abierta lo componen toda una sucesión de préstamos de alguna lengua extranjera, principalmente anglicismos, por ejemplo: бонус - bопиs, рецессия - recesión y otros muchos de contenido semejante. Ellos reflejan, ante todo, la tendencia a la internacionalización de la terminología económica, lo que se constata, por absoluta necesidad de comunicación de manera especial en el mundo de las empresas, dada la presencia constante de contactos directos y naturales con socios extranjeros. Al mismo tiempo la necesidad de un servicio de traducción altamente cualificada aumenta considerablemente.

La calidad de esta traducción depende de la competencia del traductor y su habilidad para poner al día sus conocimientos. De ahí arranca asimismo la necesidad de llevar a cabo toda una serie de estudios más profundos sobre la terminología económica y su funcionamiento real. Los diccionarios se han venido conformando con el hecho de registrar solo los términos ya asentados, los que han hecho ya fuerza de ley, sin precisar el carácter específico de su verdadero uso, que a veces va incluso más lejos de lo previsto en las propias entradas.

En lo que se refiere al aspecto práctico, esta precisión, exigible a todas luces, se hace más importante para los traductores e intérpretes, puesto que participan activamente en el proceso de integración y globalización de la economía y de la colaboración económica internacional. El objetivo principal de los traductores es, sin duda, la garantía de comunidad y equivalencia del contenido del texto original y de su correspondiente traducción. No obstante, según los lingüistas, esto se hace difícil por falta de una identidad absoluta de los códigos de lenguaje, puesto que en general tal identidad contradice a la naturaleza de cualquier lengua [Jacobson 1959].

Lo que se indicaba anteriormente determina el tema de este primer esbozo de investigación, a saber: la especificación de los parámetros del funcionamiento de algunos términos en el discurso económico actual. El objeto de este estudio es, como venimos insistiendo, la terminología que describe el fenómeno de la crisis económica y los procesos ligados a ella. En el presente trabajo se ha llevado a cabo una tentativa de seguimiento y descripción del funcionamiento de los términos dados en el discurso económico, tanto oral como escrito. Dicha descripción tiene un carácter comparativo y se hace sobre los ejemplos de tres idiomas (español, ruso e inglés), lo que nos da 
la posibilidad de seguir el espectro y la frecuencia de los lexemas estudiados en el discurso oral y escrito en las tres lenguas así como el nivel de su equivalencia.

El material de base de la presente investigación viene representado por 75 textos escritos, de los cuales 25 han sido en español, 25 en inglés y otros tantos en ruso. La cantidad del material sonoro está compuesta por 53 horas de grabación de las lenguas anteriormente citadas y han servido de fuentes de inspiración determinados sitios web del mayor prestigio, como: El servicio ruso de la Corporación Británica de radiodifusión, $\mathrm{BBC}$ news, las noticias económicas y financieras en www.newseconomy. ru, la versión electrónica del canal Noticias, etc. Cabe señalar que se han utilizado para la elaboración de este corpus publicaciones del mayor rigor científico: anuarios, revistas etc., que recogen noticias sobre la situación económica en el mundo con una referencia concreta a la crisis económica.

El objetivo de la presente investigación es la definición y estudio del espectro léxico de los términos indicados, es decir su representación como lexemas concretos, lo mismo que la frecuencia de su uso en las citadas lenguas. Ello va a permitir no solo reflexionar sobre la parte lingüística de la cuestión, sino también sacar conclusiones que contribuyan a la solución de problemas concretos de traducción e interpretación.

Los estudios teóricos sobre esta cuestión han demostrado que la investigación de tal fenómeno como discurso ha tropezado con interpretaciones varias y comentarios divergentes, lo que, a fin de cuentas, ha degenerado en un concepto demasiado turbio. Hoy día la definición más popular y menos vulnerable, según los científicos, podría, a nuestro modo de ver, ser la siguiente - "el habla sumida en la vida" (Arutiunova 1990: 137). Los lingüistas diferencian también los conceptos de discurso y de texto, considerándolos correlacionados, pero no idénticos. Lo certifica la variación de tipología del discurso y del texto en literatura teórica (Borisova 2005, Solganik 1997). Además, los científicos reconocen unívocamente la división del discurso en las variantes oral y escrita, lo que refleja el grado de complejidad de construcción y percepción de ambas. Esto conlleva, a su vez, ciertas diferencias, aunque las mismas no sean sorprendentes; por ejemplo, en el uso de unas u otras denominaciones para definir un concepto.

Dependiendo de la esfera de la comunicación humana en la que se utiliza, el discurso se agrupa en usos mucho más limitados y concretos. Uno de dichos usos o variantes es el discurso económico que atiende, sobre todo, a la esfera de las relaciones económicas y que se caracteriza por una cierta saturación de la terminología correspondiente (lo cual incluye no solo las formaciones estrictamente reglamentadas, sino también múltiples unidades de uso común, que desarrollan sin embargo nuevos significados). El discurso económico desarrolla asimismo, dentro de su especificidad, nuevas variantes y persigue fundamentalmente un carácter argumentativo y persuasivo. Estas características han sido, por otra parte, definidas en lo referente al léxico, atendiendo a cinco parámetros importantes: univocidad, pertenencia a un campo concreto, prioridad de la noción sobre el término, versatilidad y vocación de universalidad (López 2008). Los estudiosos opinan que tales características derivan de la orientación pragmática en la discusión sobre las razones de la situación económica 
formada, pronóstico de su posible desarrollo y elección de una determinada estrategia que se orienta al éxito final de una operación eficiente (Agarkova 2001).

El discurso económico se produce de dos formas - oral y escrita - que, necesariamente, presentan algunas diferencias. La variante escrita se caracteriza por un mayor nivel de complejidad en sus formulaciones, por la saturación de términos, por el estatismo y la fijación de todo lo que refleja el desarrollo de la economía a lo largo de su historia. Al contrario, el discurso oral se caracteriza por el estado "online" y determina mayoritariamente solo lo que es actual para en el día de hoy. Así, por ejemplo, en relación con la oficialmente declarada salida de la crisis el concepto reconstitución económica se sustituye, entre otros eufemismos más o menos exitosos, por programas de desarrollo e innovación.

El análisis de los materiales empíricos ha permitido sacar a luz fenómenos de mayor frecuencia que describen la situación de la crisis económica y alguno de los aspectos ligados a la misma, en las tres lenguas de referencia. Así, encontramos los siguientes conceptos: recesión, crisis económica, reconstitución de la economía / medidas de recuperación, política de precios (su incremento o disminución), mercado, crisis del presupuesto, indicadores del estado de la economía.

Estudiemos, como ejemplo práctico, los fenómenos, a nuestro entender, más ilustrativos, a saber: recesión, crisis económica y reconstitución económica.

En el discurso escrito ruso, para definir el fenómeno de recesión, se utilizan lexemas tales como рецессия - recesión; спад - caída, período recesivo, снижение - bajada, rebaja, disminución. En el discurso oral la tendencia hacia el uso del préstamo recesión es mucho más frecuente que el uso de algunas palabras, incluso bastante más claras para cualquier persona, hasta para las medianamente informadas, como caída, bajada.

El discurso escrito de la lengua inglesa muestra una serie de términos correlativos: recession - recesión, slowdown- disminución, downturn - recesión, caída; downfall - ruina, perdición; breakdown - rotura, fallo. Dichos términos se combinan frecuentemente con caracterizaciones como global y downturn. Los menos frecuentes son los lexemas slowdown, downfall, breakdown.

El discurso oral y escrito de la lengua española utiliza en el corpus de referencia y casi de manera exclusiva, una sola denominación: recesión; aunque también es cierto que en el discurso escrito aparecen las siguientes denominaciones en situaciones semejantes: caída y depresión.

En lo que se refiere al concepto de crisis económica, en la lengua rusa, tanto en el discurso escrito como en el oral, dicho concepto está representado por tres denominaciones, a saber: кризис - сrisis, экономический коллапс - colapso есопо́mico у экономическая нервозность - ataxia есопо́тіса.

Por lo que hace al discurso escrito en inglés, este nos muestra un abanico léxico mucho más extenso sobre el fenómeno que nos ocupa; así aparecen: crisis -crisis, crunch - crisis, dificultad; collapse - colapso, financial disaster - catástrofe financiera. 
El discurso oral en español está representado por tres denominaciones que aportan grados a la referencia espacial: crisis, crisis mundial, crisis económica. Aparte de las denominaciones que se usan en el discurso oral, el discurso escrito tiene más correspondencias algunas de las cuales serian: crisis financiera, crisis global y crisis fiscal. Seria interesante considerar en este caso también toda una serie de eufemismos, muchos de ellos realmente imaginativos que tratan de ocultar el término, quizá maldito, de crisis. Entre otros hemos encontrado los de situación ciertamente difícil y complicada, condiciones adversas, una coyuntura económica claramente adversa, brusca desaceleración, deterioro del contexto económico, ajuste, empeoramiento, escenario de crecimiento debilitado, periodo de serias dificultades, debilidad del crecimiento económico, difícil momento coyuntural, empobrecimiento del conjunto de la sociedad, gravedad de la situación, las cosas van claramente menos bien.

En los textos rusos, el concepto reconstitución de la economía viene también representado por otras variantes. Pero en cuanto a su diferencia con las opciones anteriores, aquí descubrimos un número sensiblemente mayor de denominaciones, lo que, al parecer, podría explicarse por los intentos activos de la comunidad de naciones para salir de la situación de crisis de la forma más rápida posible. Así, en el discurso escrito, podemos seguir el uso de estructuras más complejas, compuestas y abiertas que en el discurso oral, tales comо: стимулирование экономического роста - estimulación del crecimiento есопо́тісо, изменить ситуацию в мировой экономике - cambiar la situación de la economía mundial, изменить экономическую ситуацию - cambiar la situación económica (se supone que para bien). En el discurso oral hay variantes con una cierta transferencia metafórica: спасение глобальной экономики - salvamento de la economía global, оживить экономику - reanimar la economía у otras del mismo tenor.

En la lengua inglesa se usan, en muchos casos, combinaciones de verbos con el sustantivo economía en la posición de complemento: to lift (alzar) the economy, revitalize (revitalizar) the economy, to revive (reavivar) the economy, to boost (fortalecer) the economy, y también combinaciones con los sustantivos revival (renacimiento) y recovery (recuperación), además de otras con el adjetivo economic (económico): economic revival, economic recovery. En el lenguaje oral se encuentran solo algunas variantes semejantes: to restore (restaurar) the economy, economic recovery (point). A nuestro entender, este aspecto puede ser explicado por el propio carácter de la comunicación oral; de manera habitual, la misma se verá menos sobrecargada con unidades compuestas y preferirá entonces lanzarse hacia un régimen de austeridad y de claridad.

El discurso escrito español utiliza numerosas denominaciones, de las que las más importantes serían: recuperación, recuperar la economía, (encontrar) un remedio global, resurgimiento de la economía, crecimiento mundial, crecimiento en la economía, solución a la crisis y sacar la economía de la crisis económica. Mas, aunque aparezcan todas esas posibilidades, en el discurso oral encontraremos, lo más a menudo, solamente recuperación, crecimiento mundial y crecimiento de la economía.

En conclusión cabe señalar que la comparación de los datos del funcionamiento del término en el discurso oral y escrito resulta fecunda para descubrir la frecuencia 
de su uso en el lenguaje oral y, por lo mismo, en el establecimiento de la correlación de conceptos sinónimos, o sea de su funcionamiento activo. El discurso oral nos permite entonces seguir el funcionamiento "vivo", activo de los términos, lo que no se ha registrado aún en los diccionarios, pero que ya presenta una realidad. Este aspecto debe ser tenido muy en cuenta por los traductores e intérpretes a la hora d encarar cualquier tipo de discurso económico, ya sea oral o escrito, para evitar desviarse, en algunos casos hasta peligrosamente, del significado real del término o concepto que intentan traducir.

\section{BIBLIOGRAFÍA}

Arutunova, N. D. (1990): Diskurso, Enciclopedia lingüística, M. Sov, Enciclopedia, pp. 136-137.

Belousova, V. A., López, González, H., Raytarovskyi, V. W. (2005): Diccionario rusoespañol / español-ruso de términos económicos, Moscú, Russki Yasik.

Jacobson, R. (1959): On Linguistic Aspects of Translation, On Translation. - Cambridge, Harvard University Press: pp. 232-239.

Kandelaki, T. L. (1977): Semántica y motivación de términos, M.,Nauta.

Kashkin, V. B. (2004): Diskurso: material didáctico, Voronezh, Universidad Estatal de Voronezh.

López González, H. (2008): “Acercamiento al lenguaje económico-empresarial”. III Conferencia Internacional de Hispanistas de Rusia, Moscú, Universidad Lingüística de Moscú, Universidad de Cádiz.

López González, H. (2003): Manual práctico de lengua española aplicada a la economía, (Programa TEMPUS), León, Universidad de León.

Solganik, G. Ya. (1977): Estilística del texto, M. Flinta - Ciencia.

\section{FUENTES}

Russian service of British Broadcasting Corporation

El servicio Ruso de la corporación Británica de radiodifusión

http://www.bbc.co.uk/russian.

BBC news http://www.bbc.co.uk/.

Noticias económicas y financieras en www.newseconomy.ru

Versión electrónica del canal Noticias www.vesti.ru

http://www.elpais.com/economia/

http://www.elmundo.es/ 